\title{
THE CATEGORY OF REPRESENTATIONS OF A COMPLETELY O-SIMPLE SEMIGROUP
}

\author{
D. B. McALISTER \\ (Received 21 November 1968) \\ Communicated by G. B. Preston
}

\begin{abstract}
A. H. Clifford [1], [2] has shown that all finite dimensional representations of a completely 0 -simple semigroup $S$ over a field $\Phi$ can be obtained as extensions of those of its maximal subgroups and has given a method for constructing all such representations. This representation theory depends strongly on the fact that the representations under consideration are finite dimensional and is not adequate to deal with the infinite dimensional case or with representations over arbitrary rings. In order to determine the structure of the (contracted) algebra $\Phi(S)$ of $S$ modulo its radical, one has to consider representations which are not finite dimensional or over fields; c.f. [6]. Hence Clifford's theory does not suffice for this purpose.
\end{abstract}

In this paper we give a representation theory for a class of rings which we call Munn rings. These rings are infinite dimensional analogs of the Munn algebras considered in [9], Section 4; thus the algebra of any completely 0 -simple semigroup is a Munn ring. The form of the representations which we obtain is simpler than that obtained by Clifford yet the theory does not require that the representations should be finite dimensional or over a field.

Although the theory given here was developed to solve the problem of determining the structure of the algebra of a completely 0 -simple semigroup modulo its radical, it has proved useful in several ways. Let $S$ be an arbitrary semigroup and let $\Gamma: S \rightarrow \operatorname{Hom}(V, V)$ be an irreducible representation of $S$, where $V$ is a finite dimensional vector space over a field $\Phi$. Then it is shown in [5] that $\Gamma$ is the 'extension' of an irreducible representation $\Gamma^{*}: C_{\Gamma} \rightarrow \operatorname{Hom}(V, V)$ where $C_{\Gamma}$ is a completely 0 -simple semigroup associated with $S$ and $\Gamma$. To give an explicit construction for $\Gamma$ from $\Gamma^{*}$ one has to make use of the fact that $\Gamma^{*}\left(\Phi\left(C_{\Gamma}\right)\right)$ contains the identity $1_{V}$ of $\operatorname{Hom}(V, V)$. Proposition 2.9 of this paper gives a simple method for finding $E \in \Phi\left(C_{\Gamma}\right)$ such that $\Gamma^{*}(E)=1_{V}$ and hence for giving an explicit formula for $\Gamma$. The theory can also be used to give short proofs of some known results; Theorem 2.7 here gives a short proof of [5], Theorem 3.12 and [4], Theorem 2.1 .

As the title indicates, the approach adopted in this paper is that of studying 
the category of representations of a completely 0 -simple semigroup $S$. Thus, in Section 2, we consider the objects of the category, the representations; the main theorem of the section gives the structure of all such representations. In Section 3 , we consider the morphisms of the category. The main theorem of this section shows that the category of basic representations of $S$ is equivalent to the category of proper representations of any of the maximal subgroups of $S$. From this theorem one can deduce, as corollaries, Theorems 5.48, 5.50 and 5.51 of [3]. In Section 4 we show how the theory given in [1], [2], and related results of Lallement and Petrich [4] and Munn [9], fits in with the theory given here.

\section{Preliminaries}

In this paper all modules and vector spaces are left modules and vector spaces.

Let $\mathscr{A}$ be the category of modules over a ring $\mathfrak{U}$ with identity and let $A$ be an object in $\mathscr{A}$. If $I, A$ are sets we shall denote by $A^{I}$ the product $\mathfrak{A}$-module of $I$ copies of $A$ and by $A$ the coproduct $\mathfrak{A}$-module of $A$ copies of $A$. The appropriate projections and injections are denoted respectively by $\pi_{i}, q_{\lambda}$.

Each morphism $P:{ }^{A} A \rightarrow A^{I}$ of $\mathfrak{A}$-modules determines a set of morphisms $p_{\pi i}=q_{\lambda} P_{\pi i}: A \rightarrow A$ and conversely, given any set of morphisms $p_{\lambda i}: A \rightarrow A$, $\lambda \in A, i \in I$, there is a unique morphism $P:{ }^{\Lambda} A \rightarrow A^{I}$ of $\mathfrak{A}$-modules such that $p_{\lambda i}=q_{\lambda} P_{\pi i}$ for each $i \in I, \lambda \in A$. Thus there is a one-to-one correspondence between $\operatorname{Hom}\left({ }^{A} A, A^{I}\right)$ and the set of all $A \times I$ matrices over $\operatorname{Hom}(A, A)$. It is easily verified that this one-to-one correspondence is in fact an isomorphism of additive abelian groups.

On the other hand, given any morphism $x: A \rightarrow A$ and $(i, \lambda) \in I \times A$, there is a morphism $(x ; i, \lambda): A^{I} \rightarrow{ }^{A} A$ defined by $(x ; i, \lambda)=\pi_{i} \times q_{\lambda}$. The subgroup of Hom $\left(A^{I},{ }^{A} A\right)$ generated by all such morphisms will be denoted by $\mathscr{M}(A ; I, \Lambda)$. There is an obvious isomorphism between $\mathscr{M}(A ; I, A)$ and the additive group of finitely non-zero $I \times A$ matrices over $\operatorname{Hom}(A, A)$.

Definition 1.1. Let $\mathfrak{A}$ be a ring with identity and let $I, A$ be sets, let $P:{ }^{\Lambda} \mathfrak{U} \rightarrow \mathfrak{A}^{I}$ be a morphism of $\mathfrak{A}$-modules such that, for some $i \in I, \lambda \in \Lambda, p_{\lambda i}$ is invertible, regarded as a morphism of $\mathfrak{U}$-modules. Then the $I \times \Lambda$ Munn ring $\mathscr{M}=\mathscr{H}(\mathfrak{A} ; I, A ; P)$ over $\mathfrak{U}$ with multiplier $P$ is the ring obtained from the abelian group $\mathscr{M}(\mathfrak{U} ; I, \Lambda)$ be defining the product of $X$ and $Y$ to be $X P Y$. (Since multiplication of morphisms in the category of $\mathfrak{A}$-modules is associative and distributes over addition, $\mathscr{M}$ is a ring.)

Since there is an isomorphism between the ring $\mathfrak{A}$ and the ring Hom $(\mathfrak{A}, \mathfrak{A})$ of $\mathfrak{A}$-module endomorphisms we can identify $\mathfrak{A}$ and Hom $(\mathfrak{A}, \mathfrak{A})$. With this identification, $\mathscr{M}(\mathfrak{U} ; I, \Lambda ; P)$ is isomorphic to the ring of finitely non-zero $I \times A$ matrices over $\mathfrak{Q}$ with

$$
X \cdot Y=X P Y
$$


where $P$ is a $A \times I$ matrix over $\mathfrak{A}$ with at least one invertible entry. This leads to the following fundamental example.

EXAMPLE 1.2. Let $S=\mathscr{M}^{0}(G: I, \Lambda ; P)$ be a completely 0 -simple semigroup ([3], page 76) and let $\mathfrak{A}$ be a ring with identity. Then the (contracted) semigroup ring of $S$ over $\mathfrak{A}$ is isomorphic to the Munn ring $\mathscr{M}(\mathfrak{H}(G) ; I, \Lambda ; P)$ where $P$ is regarded as a morphism of $\mathfrak{A}(G)$ modules ${ }^{A} \mathfrak{A}(G) \rightarrow \mathfrak{A}(G)^{I}$.

If $I, \Lambda$ are finite with, say, $m$ and $n$ members respectively then we denote $\mathscr{M}(\mathfrak{H} ; I, \Lambda ; P)$ by $\mathscr{M}(\mathfrak{H} ; m, n ; P)$. In the cases when $\mathfrak{A}$ is the group ring of a finite group and each non-zero entry in $P$ belongs to the group, such Munn rings are precisely the semigroup rings of finite 0 -simple semigroups.

Definition 1.3. Let $\mathfrak{A}$ be a ring and $\mathfrak{N}$ a ring with identity. Then a representation $\Gamma$ of $\mathfrak{A}$ over $\mathfrak{N}$ is a morphism of the ring $\mathfrak{A}$ into the ring Hom $(C, C)$ of endomorphisms of an $\mathfrak{R}$-module $C$.

A morphism $\psi: \Gamma \rightarrow \Delta$ between representations $\Gamma: \mathfrak{A} \rightarrow \operatorname{Hom}(C, C)$ and $\Delta: \mathfrak{A} \rightarrow \operatorname{Hom}(D, D)$ of $\mathfrak{U}$ over $\mathfrak{N}$ is a morphism $\psi: C \rightarrow D$ of $\mathfrak{R}$-modules such that

for each $x \in \mathfrak{A}$.

$$
\Gamma(x) \psi=\psi \Delta(x)
$$

When morphisms between representations are defined in this way, it is clear that the representations of $\mathfrak{A}$ over $\mathfrak{A}$ and their morphisms constitute a category. This category $R(\mathfrak{H}, \mathfrak{R})$ is equivalent to the category of all left $\mathfrak{R}$-right $\mathfrak{A}$ bimodules; [10], p. 115. Hence $R(\mathfrak{A}, \mathfrak{N})$ is a complete and cocomplete abelian category; cf [8], p. 69. Further the epics and monics are precisely those epic and monic morphisms of $\mathfrak{N}$-modules which belong to $R(\mathfrak{H}, \mathfrak{R})$.

As a point of notation, we shall usually write representations to the left of their argument: thus $\Gamma(x)$. However, in general, we shall follow the diagrammatic convention with regard to the composition of morphisms; if $f: A \rightarrow B, g: B \rightarrow C$ then $f g$ is the composite morphism $A \rightarrow C$.

Definition 1.4. For each representation $\Gamma: \mathfrak{A} \rightarrow \operatorname{Hom}(C, C)$ of a ring $\mathfrak{A}$ over a ring $\mathfrak{R}$ with identity we define

$$
\begin{aligned}
N=N(\Gamma) & =\{c \in C: c \Gamma(x)=0 \text { for each } x \in \mathfrak{A}\}, \\
I & =I(\Gamma)=\text { submodule generated by }\{c \Gamma(x): c \in C, x \in \mathfrak{A}\} .
\end{aligned}
$$

The representation $\Gamma$ is a null representation if $N(\Gamma)=C$ or, equivalently, if $I(\Gamma)=0 ; \Gamma$ is a basic representation if $N(\Gamma)=0$ and $I(\Gamma)=C$.

The representation $\Gamma$ is irreducible if it has no non-trivial subrepresentations. Thus $\Gamma$ is irreducible if and only if when

$$
0 \rightarrow \Gamma_{1} \rightarrow \Gamma \rightarrow \Gamma_{2} \rightarrow 0
$$

is an exact sequence of representations either $\Gamma$ and $\Gamma_{1}$ are equivalent or $\Gamma$ and $\Gamma_{2}$ are equivalent. Likewise $\Gamma$ is indecomposable if, when 


$$
0 \rightarrow \Gamma_{1} \rightarrow \Gamma \rightarrow \Gamma_{2} \rightarrow 0
$$

is a split exact sequence, either $\Gamma$ and $\Gamma_{1}$ are equivalent or $\Gamma$ and $\Gamma_{2}$ are equivalent. Thus $\Gamma$ is indecomposable if when

$$
\Gamma=\Gamma_{1} \oplus \Gamma_{2}
$$

either $\Gamma_{1}=0$ or $\Gamma_{2}=0$. As a slightly weaker condition on $\Gamma$ we define $\Gamma$ to be proper if

$$
\Gamma=\Gamma_{1} \oplus \Gamma_{2}
$$

with $\Gamma_{1}$ null implies $\Gamma_{1}=0$.

Clearly every irreducible representation is basic and every basic representation is proper. Neither converse holds in general; however, if the ring $\mathfrak{A}$ has an identity, each proper representation is basic. Thus to find the irreducible representations of a ring $\mathfrak{A}$ it suffices to consider the basic representations. The following proposition shows that with any representation of a Munn ring there is associated a unique basic representation.

Proposition 1.5. Let $\mathfrak{A}$ be a ring which is generated, as a group, by $\mathfrak{A}^{2}$. For each representation $\Gamma: \mathfrak{A} \rightarrow \operatorname{Hom}(C, C)$ of $\mathfrak{A}$ over a ring $\mathfrak{R}$ with identity, there is a sequence

$$
\Gamma_{1} \rightarrow \Gamma_{2} \rightarrow \Gamma
$$

of subrepresentations such that $\Gamma_{1}$ and $\Gamma / \Gamma_{2}$ are null and $\Gamma_{2} / \Gamma_{1}$ is basic. The basic representations determined by any two such sequences of subrepresentations of $\Gamma$ are equivalent.

Proof. Let $N(\Gamma)=N$ and $I(\Gamma)=I$ be as in Definition 1.4. Then clearly $N$ and $I+N$ are invariant under $\Gamma$; denote by $\Gamma_{1}$ and $\Gamma_{2}$ the restrictions and corestrictions of $\Gamma$ to $N$ and $I+N$ respectively. Then certainly $\Gamma_{1}$ and $\Gamma / \Gamma_{2}$ are null so that we need only show that $\Gamma_{2} / \Gamma_{1}=\Gamma^{*}$ is basic.

Now $\Gamma^{*}$ acts on $C^{*}=(I+N) / N$ by

$$
(N+u) \Gamma^{*}(x)=N+u \Gamma(x)
$$

for each $u \in I, x \in \mathfrak{U}$. Thus

$$
N\left(\Gamma^{*}\right)=\{N+u: u \Gamma(x) \in N \text { for each } x \in \mathfrak{A}\} .
$$

If $u \Gamma(\mathfrak{C}) \subseteq N$ then $u \Gamma\left(\mathfrak{U}^{2}\right)=0$ and so, since $\mathfrak{A}$ is generated as a group by $\mathfrak{A}^{2}$, $u \Gamma(\mathfrak{A})=0$. It follows that $N\left(\Gamma^{*}\right)=N$ the zero of $C^{*}$. Further

$$
I\left(\Gamma^{*}\right)=\text { submodule generated by }\{N+u \Gamma(x): u \in I, x \in \mathfrak{A}\} .
$$

Since $\mathfrak{A}$ is generated by $\mathfrak{A}^{2}$ it follows that

$$
I=\text { submodule generated by }\{u \Gamma(x): u \in I, x \in \mathfrak{A}\} .
$$

Hence $I\left(\Gamma^{*}\right)=(N+I) / N=C^{*}$. Therefore $\Gamma^{*}$ is basic. 
Conversely, suppose that $\Delta_{1} \rightarrow \Delta_{2} \rightarrow \Gamma$ is a sequence of subrepresentations of $\Gamma$ such that $\Delta_{1}$ and $\Gamma / \Delta_{2}$ are null while $\Delta_{2} / \Delta_{1}$ is basic. Let $N_{1}$ denote the submodule on which $\Delta_{1}$ acts and let $I_{1}$ denote that on which $\Delta_{2}$ acts.

Since $\Delta_{1}$ is null, we clearly have $N_{1} \subseteq N$ so that $N_{1} \subseteq N \cap I_{1}$. On the other hand, if $u \in N \cap I_{1}$ then

$$
\left(N_{1}+u\right) \Delta_{2} / \Delta_{1}(x)=N_{1}+u \Gamma(x)=N_{1}
$$

for each $x \in \mathfrak{A}$. Hence, since $\Delta_{2} / \Delta_{1}$ is basic, $u \in N_{1}$. Thus we have

$$
N_{1}=N \cap I \text {. }
$$

Since $\Gamma / \Delta_{2}$ is null, we also have $I \subseteq I_{1}$ so that $I+N_{1} \subseteq I_{1}$. On the other hand, since $\Delta_{2} / \Delta_{1}$ is basic,

$$
I_{1} / N_{1}=\left[\left(I_{1} / N_{1}\right) \Delta_{2} / \Delta_{1}(\mathfrak{A})\right]=\left[N_{1}+I_{1} \Gamma(\mathfrak{H})\right] / N_{1} \subseteq\left(N_{1}+I\right) / N_{1}
$$

so that $I_{1} \subseteq I+N_{1}$. Hence

$$
I_{1}=I+N_{1} .
$$

Define $\psi: I_{1} / N_{1}=\left(I+N_{1}\right) / N_{1} \rightarrow(I+N) / N$ by $\left(N_{1}+u\right) \psi=N+u$. Then since $N_{1} \subseteq N, \psi$ is a well defined morphism of $I_{1} / N_{1}$ onto $(I+N) / N$. If $\left(N_{1}+u\right) \psi=N$ then $u \in I_{1} \cap N=N_{1}$ so $\psi$ is also one-to-one; thus $\psi$ is an isomorphism. Finally, for each $u \in I_{1}, x \in \mathfrak{A}$,

$$
\left(N_{1}+u\right) \Delta_{2} / \Delta_{1}(x) \psi=N+u \Gamma(x)=(N+u) \Gamma^{*}(x)=\left(N_{1}+u\right) \psi \Gamma^{*}(x) .
$$

Hence $\psi$ is an equivalence $\Delta_{2} / \Delta_{1} \rightarrow \Gamma^{*}$.

COROLlaRY 1.6. For each representation $\Gamma$ of a Munn ring $\mathscr{M}=\mathscr{M}(\mathfrak{A} ; I, \Lambda ; P)$ over a ring $\mathfrak{R}$ with identity there is a sequence

$$
\Gamma_{1} \rightarrow \Gamma_{2} \rightarrow \Gamma
$$

of subrepresentations such that $\Gamma_{1}$ and $\Gamma / \Gamma_{2}$ are null while $\Gamma_{2} / \Gamma_{1}$ is basic.

Proof. Pick $(i, \lambda) \in I \times \Lambda$ such that $p_{\lambda i}$ is invertible. Then

$$
(x ; j, \mu)=(x ; j, \lambda)\left(p_{\lambda i}^{-1} ; i, \mu\right)
$$

for each $(x ; j, \mu) \in \mathscr{M}$. Hence, since $\mathscr{M}$ is generated as a group by the $(x ; j, \mu)$, it is generated by $\mathscr{M}^{2}$ and the proposition applies.

REMARK 1.7. If $\psi: \Gamma \rightarrow \Delta$ is a morphism between representations of a ring then $\psi$ maps $N(\Gamma)$ into $N(\Delta)$ and $I(\Gamma)$ into $I(\Delta)$. Hence, under the conditions of Proposition 1.5, $\psi$ gives rise to a morphism $\psi^{*}: \Gamma^{*} \rightarrow \Delta^{*}$ of basic representations. This correspondence is a functor from $R(\mathfrak{A}, \mathfrak{N})$ to the full subcategory $B(\mathfrak{A}, \mathfrak{R})$ of basic representations of $\mathfrak{A}$ over $\mathfrak{R}$. 


\section{Representations of Munn rings}

Let $\gamma: \mathfrak{A} \rightarrow \operatorname{Hom}(A, A)$ be a representation of a ring $\mathfrak{A}$, with identity, over a ring $\mathfrak{N}$ with identity and let $I, \Lambda$ be sets. Corresponding to each morphism $P:{ }^{A} \mathfrak{U} \rightarrow \mathfrak{U}^{I}$ obtained from a set of commutative diagrams

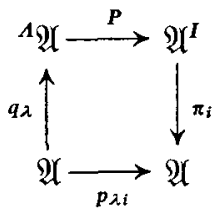

there is a unique morphism $\gamma(P):{ }^{A} A \rightarrow A^{I}$ of $\mathfrak{R}$-modules obtained from the commutative diagrams

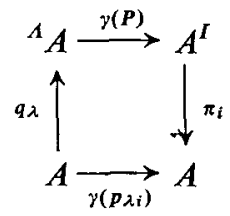

( $q_{\lambda}, \pi_{i}$ are used here to denote the appropriate injections and projections).

Similarly, corresponding to each $X \in \mathscr{M}(\mathfrak{H} ; I, \Lambda)$ there is a unique $\gamma(X): A^{I} \rightarrow{ }^{\wedge} A$.

Suppose that $\Gamma: \mathscr{M} \rightarrow \operatorname{Hom}(C, C)$ is a representation of a Munn ring $\mathscr{M}=\mathscr{M}(\mathfrak{H} ; I, \Lambda ; P)$ over a ring $\mathfrak{N}$ with identity. Then, for each $(\lambda, i) \in \Lambda \times I$ such that $p_{\lambda i}$ is invertible, we obtain a proper representation $\gamma_{i \lambda}$ of $\mathfrak{A}$ over $\mathfrak{R}$ as follows.

Let $C_{i \lambda}=C \Gamma\left(p_{\lambda i}^{-1} ; i, \lambda\right)$; then $C_{i \lambda}$ is an $\mathfrak{R}$-submodule of $C$. Further, since, for each $x \in \mathfrak{A}$,

$$
\begin{aligned}
& \left(x p_{\lambda i}^{-1} ; i, \lambda\right)=\left(x p_{\lambda i}^{-1} ; i, \lambda\right)\left(p_{\lambda i}^{-1} ; i, \lambda\right) \\
& C \Gamma\left(x p_{\lambda i}^{-1} ; i, \lambda\right) \subseteq C_{i \lambda}
\end{aligned}
$$

also for each $x \in \mathfrak{A}$. Define $\gamma_{i \lambda}: \mathfrak{A} \rightarrow$ Hom $\left(C_{i \lambda}, C_{i \lambda}\right)$ by setting

$$
\gamma_{i \lambda}(x)=\text { restriction and corestriction of } \Gamma\left(x p_{\lambda i}^{-1} ; i, \lambda\right) \text { to } C_{i \lambda} .
$$

Then $\gamma_{i \lambda}$ is a representation of $\mathfrak{A}$ over $\mathfrak{R}$ and, since $\gamma_{i \lambda}(e)$ is the identity on $C_{i \lambda}$, $\gamma_{i \lambda}$ is proper.

Definition 2.1. $\gamma_{i \lambda}: \mathfrak{A} \rightarrow \operatorname{Hom}\left(C_{i \lambda}, C_{i \lambda}\right)$ is the representation of $\mathfrak{A}$ induced by $\Gamma, i, \lambda$.

If $\Gamma: \mathscr{M} \rightarrow \operatorname{Hom}(C, C), \Delta: \mathscr{M} \rightarrow \operatorname{Hom}(D, D)$ are representations of $\mathscr{M}$ over $\mathfrak{N}$ then, for each $\psi: \Gamma \rightarrow \Delta$,

$$
C_{i \lambda} \psi=C \Gamma\left(p_{\lambda i}^{-1} ; i, \lambda\right) \psi=C \psi \Delta\left(p_{\lambda i}^{-1} ; i, \lambda\right) \subseteq D_{i \lambda} .
$$


Hence, if $\psi_{i \lambda}$ is the restriction and corestriction of $\psi$ to a morphism $C_{i \lambda} \rightarrow D_{i \lambda}$, $\psi_{i \lambda}$ is a morphism $\gamma_{i \lambda} \rightarrow \delta_{i \lambda}$; it is the morphism induced by $\psi, i, \lambda$.

TheOREM 2.2. Let $\mathscr{M}=\mathscr{M}(\mathfrak{H} ; I, \Lambda ; P)$ be a Munn ring and let $(\lambda, i) \in \Lambda \times I$ be such that $p_{\lambda i}$ is invertible. Then the mapping which associates with each representation $\Gamma$ of $\mathscr{M}$, over a ring $\mathfrak{A}$ with identity, the representation $\gamma_{i \lambda}$ of $\mathfrak{U}$ induced by $\Gamma, i, \lambda$ and with each morphism $\psi: \Gamma \rightarrow \Delta$ of representations the morphism $\psi_{i \lambda}$ : $\gamma_{i \lambda} \rightarrow \delta_{i \lambda}$ is a limit and colimit preserving functor from $R(\mathscr{M}, \mathfrak{R})$ to the category $P(\mathfrak{A}, \mathfrak{N})$ of proper representations of $\mathfrak{A}$ over $\mathfrak{R}$.

Proof. When $\gamma_{i \lambda}$ and $\psi_{i \lambda}$ are defined as above it is clear that the mapping under consideration is a functor. It is a straightforward matter to show that this functor preserves products and intersections, coproducts and cointersections. Hence ([8], Theorem 2.4) it is limit and colimit preserving.

Theorem 2.2 shows that the representations of $\mathscr{M}$ determine representations of $\mathfrak{A}$. We now turn to the converse question. How do representations of $\mathfrak{A}$ determine representations of $\mathscr{M}$ ?

Theorem 2.3. Let $\mathscr{M}=\mathscr{M}(\mathfrak{A} ; I, \Lambda ; P)$ be a Munn ring and let $\Gamma: \mathscr{M} \rightarrow$ Hom $(C, C)$ be a representation of $\mathscr{M}$ over a ring $\mathfrak{N}$ with identity. Let $(\lambda, i) \in \Lambda \times I$ be such that $p_{\lambda i}$ is invertible and let $\gamma: \mathfrak{H} \rightarrow \operatorname{Hom}(A, A)$ be a representation of $\mathfrak{A}$ over $\mathfrak{R}$ which is equivalent to that induced by $\Gamma, i, \lambda$. Then there exist morphisms $Q:{ }^{A} A \rightarrow C, R: C \rightarrow A^{I}$ of $\Re$-modules such that

$$
Q R=\gamma(P)
$$

and such that, for each $X \in \mathscr{M}$,

$$
\Gamma(X)=R \gamma(X) Q
$$

Conversely, let $\gamma: \mathfrak{A} \rightarrow \operatorname{Hom}(A, A)$ be a proper representation of $\mathfrak{A}$ over $\mathfrak{R}$ and let $Q:{ }^{A} A \rightarrow C, R: C \rightarrow A^{I}$ be morphisms of $\mathfrak{R}$-modules such that (2.1) is satisfied. Then $\Gamma: \mathscr{M} \rightarrow \operatorname{Hom}(C, C)$ defined by (2.2) is a representation of $\mathscr{M}$ over $\mathfrak{\Re}$. Further, for each $(\lambda, i) \in \Lambda \times I$ such that $p_{\lambda i}$ is invertible, the representation $\gamma_{i \lambda}: \mathfrak{A} \rightarrow \operatorname{Hom}\left(C_{i \lambda}, C_{i \lambda}\right)$ of $\mathfrak{A}$ induced by $\Gamma, i, \lambda$ is equivalent to $\gamma$.

Proof. For each $(x ; j, \mu) \in \mathscr{M}$,

$$
(x ; j, \mu)=\left(p_{\lambda i}^{-1} ; j, \lambda\right)\left(x p_{\lambda i}^{-1} ; i, \lambda\right)(e ; i, \mu)
$$

where $e$ is the identity of $\mathfrak{A}$. Thus

$$
\Gamma(x ; j, \mu)=\bar{R}_{j} \Gamma\left(x p_{\lambda i}^{-1} ; i, \lambda\right) \bar{Q}_{\mu}
$$

where $\bar{R}_{j}=\Gamma\left(p_{\lambda i}^{-1} ; j, \lambda\right)$ and $\bar{Q}_{\mu}=\Gamma(e ; i, \mu)$. Since $\left(p_{\lambda i}^{-1} ; i, \lambda\right)$ is a right identity for $\left(p_{\lambda i}^{-1} ; j, \lambda\right)$, the range of $\bar{R}_{j}$ is contained in $C_{i \lambda}$. Let $\alpha: C_{i \lambda} \rightarrow A$ be the equivalence between $\gamma_{i \lambda}$ and $\gamma$; denote by $Q_{\mu}$ the composite of $\alpha^{-1}$ and the restriction of $\bar{Q}_{\mu}$ to $C_{i \lambda}$ and by $R_{j}$ the composite of the corestriction of $\bar{R}_{j}$ to $C_{i \lambda}$ and $\alpha$. Then 


$$
\begin{aligned}
\Gamma(x ; j, \mu) & =\bar{R}_{j} \Gamma\left(x p_{\lambda i}^{-1} ; i, \lambda\right) \bar{Q}_{\mu} \\
& =R_{j} \gamma(x) Q_{\mu} .
\end{aligned}
$$

Let $Q$ be the unique morphism ${ }^{A} A \rightarrow C$ such that $q_{\mu} Q=Q_{\mu}$ for each $\mu \in A$ and let $R$ be the unique morphism $C \rightarrow A^{I}$ such that $R \pi_{j}=R_{j}$ for each $j \in I$. Then

$$
\Gamma(x ; j, \mu)=R \gamma(x ; j, \mu) Q
$$

for each $(x ; j, \mu) \in \mathscr{M}$. Extend $\Gamma$ by additivity. Then

$$
\Gamma(X)=R \gamma(X) Q
$$

for each $X \in \mathscr{M}$ whence (2.2) holds.

For each pair $(j, \mu) \in I \times \Lambda, Q_{\mu} R_{j}=\alpha^{-1} U_{\mu} V_{j} \alpha$ where $U_{\mu}$ is the restriction of $\bar{Q}_{\mu}$ to $C_{i \lambda}$ and $V_{j}$ is the corestriction of $\bar{R}_{j}$ to $C_{i \lambda}$. Since $\bar{Q}_{\mu} \bar{R}_{j}=\Gamma\left(p_{\mu j} p_{\lambda i}^{-1} ; i, \lambda\right)$ it follows that $U_{\mu} V_{j}=\gamma_{i \lambda}\left(p_{\mu j}\right)$ and thus

$$
Q_{\mu} R_{j}=\gamma\left(p_{\mu j}\right)
$$

Hence $Q R=\gamma(P)$ and (2.1) holds.

On the other hand, if $\gamma: \mathfrak{A} \rightarrow \operatorname{Hom}(A, A)$ is a representation of $\mathfrak{A}$ over $\mathfrak{N}$ and $Q, R$ satisfy $(2.1)$, it is easy to see that $\Gamma$ defined by (2.2) is a representation of $\mathscr{M}$. Hence it remains to prove that the representation of induced by $\Gamma, i, \lambda$ is equivalent to $\gamma$.

For each $\mu \in A$, let $Q_{\mu}=q_{\mu} Q$ where $q_{\mu}$ is the $\mu^{\text {th }}$ injection $A \rightarrow^{\Lambda} A$. For each $j$, let $R_{j}=R \pi_{j}$ where $\pi_{j}$ is the $j^{\text {th }}$ projection $A^{I} \rightarrow A$. Then $Q_{\lambda} R_{i}=\gamma\left(p_{\lambda i}\right)$ which, since $\gamma$ is proper, is invertible. Hence

$Q_{\lambda}$ is monic and $R_{i}$ is epic.

By definition,

$$
\begin{aligned}
C_{i \lambda} & =C \Gamma\left(p_{\lambda i}^{-1} ; i, \lambda\right) \\
& =C R_{i} \gamma\left(p_{\lambda i}^{-1}\right) Q_{\lambda} .
\end{aligned}
$$

Hence, since $R_{i}$ is epic, $C_{i \lambda}=A \gamma\left(p_{\lambda i}^{-1}\right) Q_{\lambda}$. Let $\eta: A \rightarrow C_{i \lambda}$ be the corestriction of $\gamma\left(p_{\lambda i}^{-1}\right) Q_{\lambda}$ to $C_{i \lambda}$; since $\gamma\left(p_{\lambda i}^{-1}\right)$ and $Q_{\lambda}$ are monic, $\eta$ is an isomorphism. Consider the diagram

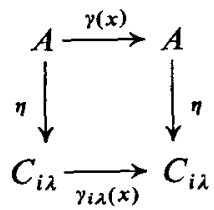

Then $\gamma(x) \eta$ is the corestriction of $\gamma\left(x p_{\lambda i}^{-1}\right) Q_{\lambda}$ to $C_{i \lambda}$ while $\eta \gamma_{i \lambda}(x)$ is the composite of $\eta$ and the restriction and corestriction of $R_{i} \gamma\left(x p_{\lambda i}^{-1}\right) Q_{\lambda}$ to $C_{i \lambda}$. But the latter is just the corestriction of 


$$
\gamma\left(p_{\lambda i}^{-1}\right) Q_{\lambda} R_{i} \gamma\left(x p_{\lambda i}^{-1}\right) Q_{\lambda}=\gamma\left(x p_{\lambda i}^{-1}\right) Q_{\lambda}
$$

to $C_{i \lambda}$. Hence the diagram commutes for each $x \in \mathscr{M}$ and thus $\gamma$ is equivalent to $\gamma_{i \lambda}$.

Theorem 2.3 reduces the problem of finding the representations of $\mathscr{M}$ to that of finding morphisms $Q:{ }^{A} A \rightarrow C$ and $R: C \rightarrow A^{I}$ such that $Q R=\gamma(P)$. The problem of constructing all such factorisations is not considered here; it has been solved by Clifford [1], [2] for finite dimensional representations over a field.

Notation 2.4. If $\gamma: \mathfrak{A} \rightarrow \operatorname{Hom}(A, A)$ is a proper representation of $\mathfrak{A}$ and $Q, R$ satisfy $(2.1)$ then we denote by $[\gamma ; Q, R]$ the representation of $\mathscr{M}=$ $\mathscr{M}(\mathfrak{A} ; I, \Lambda ; P)$ given by $(2.2)$.

Proposition 2.5. Let $\Gamma=[\gamma ; Q, R]$ be a representation of the Munn ring $\mathscr{M}(\mathfrak{A} ; I, \Lambda ; P)$. Then

(i) $N(\Gamma)=\operatorname{Ker} R$;

(ii) $I(\Gamma)=$ Image $Q$.

Proof. (i) Certainly Ker $R \subseteq N(\Gamma)$. On the other hand, if $c \notin \operatorname{Ker} R$ then $c R_{j}=c R \pi_{j} \neq 0$ for some $j \in I$. For such $j \in I, c R_{j} \gamma(e) \neq 0$ because $\gamma(e)$ is the identity morphism on $A$. There exists $(\lambda, i) \in \Lambda \times I$ such that $p_{\lambda i}$ is invertible and, by (2.3), $Q_{\lambda}$ is then monic. Hence

so that $c \notin N(\Gamma)$.

$$
c \Gamma(e ; j, \lambda)=c R_{j} \gamma(e) Q_{\lambda} \neq 0
$$

(ii) By the definition of $\Gamma, I(\Gamma) \subseteq$ Image $Q$. To obtain the converse inclusion, pick $i \in I$ such that, for some $\lambda \in \Lambda, p_{\lambda i}$ is invertible. Then

$$
C \Gamma(e ; i \mu)=C R_{i} \gamma(e) Q_{\mu}=A Q_{\mu}
$$

for each $\mu \in \Lambda$ since, by (2.3), $R_{i}$ is epic. Hence $I(\Gamma)$ contains the submodule generated by all the $A Q_{\mu}$. The latter is just the image of $Q$. Therefore Image $Q=$ $I(\Gamma)$.

COROLlary 2.6. Let $\Gamma=[\gamma ; Q, R]$ be a representation of $\mathscr{M}=\mathscr{M}(\mathfrak{A} ; I, \Lambda ; P)$, $\Gamma: \mathscr{M} \rightarrow \operatorname{Hom}(C, C)$ where $C$ is a finite dimensional vector space over a field $\Phi$. Then $\Gamma$ is proper if and only if

$$
\operatorname{dim} C=\operatorname{rank} Q+\operatorname{rank} R-\operatorname{rank} \gamma(P) .
$$

Proof. $\Gamma$ is proper if and only if $N(\Gamma) \subseteq I(\Gamma)$ which occurs if and only if Ker $R \subseteq \operatorname{Im} Q$. Since $Q R=\gamma(P)$

$$
\text { rank } \gamma(P)=\operatorname{dim} \operatorname{Im} \gamma(P)=\operatorname{dim} \operatorname{Im} Q-\operatorname{dim}(\operatorname{Im} Q \cap \operatorname{Ker} R) .
$$

Hence $\Gamma$ is proper if and only if

$$
\text { rank } \begin{aligned}
\gamma(P) & =\operatorname{rank} Q-\operatorname{dim} \operatorname{Ker} R \\
& =\operatorname{rank} Q+\operatorname{rank} R-\operatorname{dim} C .
\end{aligned}
$$


When Corollary 2.6 is applied to the algebra of a completely 0 -simple semigroup it gives the result of [1] Theorem 5.1. Likewise the next theorem shows that the basic representations as defined here coincide with those defined in [1]. It thus provides an alternative proof of [5] Theorem 3.12.

THEOREM 2.7. A representation $\Gamma=[\gamma ; Q, R]$ of $\mathscr{M}=\mathscr{M}(\mathfrak{H} ; I, \Lambda ; P)$ is basic if and only if $Q$ is epic and $R$ is monic. If $\Gamma$ is basic then

$$
\operatorname{Ker} \Gamma=\{X \in \mathscr{M}: \gamma(P X P)=0\} .
$$

Proof. $\Gamma$ is basic if and only if $I(\Gamma)=$ codomain $Q$ and $N(\Gamma)=0$. But, by Proposition 2.5, $I(\Gamma)=\operatorname{Im} Q$ and $N(\Gamma)=\operatorname{Ker} R$. Hence $\Gamma$ is basic if and only if $Q$ is epic and $R$ is monic.

If $X \in \operatorname{Ker} \Gamma$ then $R \gamma(X) Q=0$ and so

$$
\gamma(P X P)=Q R \gamma(X) Q R=0 .
$$

On the other hand $\gamma(P X P)=0$ implies

$$
Q \Gamma(X) R=Q R \gamma(X) Q \ddot{R}=\gamma(P X P)=0,
$$

and hence, because $Q$ is epic and $R$ is monic, $\Gamma(X)=0$. Thus

$$
\operatorname{Ker} \Gamma=\{X \in \mathscr{M}: \gamma(P X P)=0\} \text {. }
$$

Remark 2.8. Lallement and Petrich [4] have shown that if $\mathscr{M}$ is the algebra of a finite 0 -simple semigroup and $\Gamma$ is an irreducible representation of which extends $\gamma$ then

$$
\operatorname{Ker} \Gamma=\{X \in \mathscr{M}: \gamma(P X P)=0\} .
$$

In [5], Theorem 6.4, it is shown that the finite dimensional irreducible representations of an arbitrary semigroup $S$ over a field $\Phi$ are obtained from irreducible representations of associated completely 0 -simple semigroups. To obtain an irreducible representation $\Gamma$ of $S$ from an irreducible representation $\Gamma^{*}: T \rightarrow \operatorname{Hom}(C, C)$ of an associated completely 0 -simple semigroup, $T$ say, we need to make use of the fact that $\Gamma^{*}(\Phi(T))$ contains the identity morphism of $T$. As an application of Theorem 2.7, we give a characterisation of $X \in \Phi(T)$ such that $\Gamma^{*}(X)=1_{C}$.

Proposition 2.9. Let $\Gamma=[\gamma ; Q, R]$ be a basic representation of $\mathscr{M}=$ $\mathscr{M}(\mathfrak{A} ; I, \Lambda ; P), \Gamma: \mathscr{M} \rightarrow \operatorname{Hom}(C, C)$ over a ring $\mathfrak{N}$ with identity. Then $\Gamma(E)$ is the identity morphism on $C$ if and only if $\gamma(P)=\gamma(P E P)$, where $E \in \mathscr{M}$.

Proof. If $\Gamma(E)=1_{C}$ then $R \gamma(E) Q=1_{C}$ and so

$$
\gamma(P E P)=Q R \gamma(E) Q R=Q 1_{C} R=\gamma(P) .
$$

Conversely, if $\gamma(P E P)=\gamma(P)$, then

$$
Q(\Gamma(X) \Gamma(E)-\Gamma(X)) R=\gamma(P X P E P)-\gamma(P X P)=0
$$


and so, since $Q$ is epic and $R$ is monic, $\Gamma(X) \Gamma(E)=\Gamma(X)$ for each $X \in \mathscr{M}$. Since $I(\Gamma)=C$, it follows that $\Gamma(E)=1_{C}$.

Proposition 2.9 applies in particular to finite 0-simple semigroups $S=$ $\mathscr{M}^{0}(G ; m, n ; P)$. Suppose $\Phi$ is a field whose characteristic does not divide the order of $G$, so that $\Phi(G)$ is semisimple. Then $\Phi(S) / \operatorname{Rad} \Phi(S) \approx \Phi(S) / B(\Phi(S))$ where

$$
B(\Phi(S))=\{X \in \Phi(S): P X P=0\}
$$

([4] Theorem 2.1, [6] Theorem 3.6).

Thus $E$ in $\Phi(S)$ is such that $E+\operatorname{Rad} \Phi(S)$ is the identity of $\Phi(S) / \operatorname{Rad} \Phi(S)$ if and only if

$$
P=P E P \text {. }
$$

EXAMPLE 2.10. The regular representation of a finite 0-simple semigroup is not in general faithful. (It is faithful if and only if no two rows of the multiplier (sandwich matrix) $P$ are proportional; [3], p. 130.) However, if we consider the representation of $S$ induced by the regular representation of $S^{1}$, we get a faithful representation $\Gamma^{1}$.

If $S=\mathscr{M}^{0}(G ; m, n ; P)$ and $\Phi$ is a field then $\Phi\left(S^{1}\right)$ has a basis

$$
e_{u}, e_{m n s+1} \quad \text { where } u=(j-1) m s+(i-1) s+k
$$

$l \leqq k \leqq s, l \leqq j \leqq n, l \leqq i \leqq m$ and

$$
e_{m n s+1}=1, e_{u}=\left(x_{k} ; i, j\right) \text { with } x_{1} \text { the identity of } G .
$$

Relative to this basis $\Gamma^{1}=[\gamma ; Q, R]$ where $\gamma$ is the direct sum of $m$ copies of the regular representation of $G$

and

$$
R=\left[\begin{array}{c}
\gamma(P) \\
l_{1} \cdots l_{m 2_{s}}
\end{array}\right] \quad Q=\left[I_{m n s} 0\right]
$$

$$
l_{u}= \begin{cases}1 & \text { if } u=(i-1)(m+1) s+1 \quad 1 \leqq i \leqq m \\ 0 & \text { otherwise }\end{cases}
$$

By Corollary 2.6, $\Gamma^{1}$ is proper if and only if

$$
\begin{aligned}
\operatorname{rank} \gamma(P)+m n s+1 & =\operatorname{rank} Q+\operatorname{rank} R \\
& =m n s+\operatorname{rank} R .
\end{aligned}
$$

Hence $\Gamma^{1}$ is proper if and only if rank $R=$ rank $\gamma(P)+1$. This can occur if and only if the final row is independent of the previous ones; if this is not the case the proper part of $\Gamma^{1}$ is equivalent to the regular representation of $S$. ([7], Theorem 3.1). 


\section{Morphisms of representations}

Theorem 2.2 gives a construction for the representations of a Munn ring. In this section we consider the problem of describing the morphisms between representations of Munn rings.

Lemma 3.1. Let $\alpha: A \rightarrow B$ be a morphism of $\mathfrak{U}$-modules, where $\mathfrak{A}$ is a ring with identity, and let $I, A$ be sets. Denote by ${ }^{\wedge} \alpha$ and $\alpha^{I}$ respectively the unique morphisms ${ }^{A} A \rightarrow{ }^{A} B$ and $A^{I} \rightarrow B^{I}$ determined by $\alpha$.

(i) if $\gamma: A \rightarrow A, \delta: B \rightarrow B$ are such that $\gamma \alpha=\alpha \delta$ then

for each $i \in I, \lambda \in A$.

$$
(\gamma ; i, \lambda)^{A} \alpha=\alpha^{I}(\delta ; i, \lambda)
$$
then

(ii) if $\gamma_{i \lambda}: A \rightarrow A, \delta_{i \lambda}: B \rightarrow B$ are such that $\gamma_{i \lambda} \alpha=\alpha \delta_{i \lambda}$ for each $i \in I, \lambda \in \Lambda$

$$
\Gamma \alpha^{I}={ }_{\alpha \Delta}
$$

where $\Gamma$ is the unique morphism ${ }^{\wedge} A \rightarrow A^{I}$ determined by the $\gamma_{i \lambda}$ and $\Delta$ is the unique morphism ${ }^{A} B \rightarrow B^{I}$ determined by the $\delta_{i \lambda}$.

PROOF. The proof of this lemma is a matter of straightforward computation.

THEOREM 3.2. Let $\Gamma=[\gamma ; Q, R], \Delta=[\delta, U, V]$ be representations of a Munn ring $\mathscr{M}=\mathscr{M}(\mathfrak{U} ; I, \Lambda ; P)$ over a ring $\mathfrak{N}$ with identity. Then a morphism $\psi:$ Codomain $Q \rightarrow$ Codomain $U$ of $\mathfrak{N}$-modules is a morphism $\Gamma \rightarrow \Delta$ in $R(\mathscr{M}, \mathfrak{N})$ if and only if there is a morphism $\alpha: \gamma \rightarrow \delta$ in $P(\mathfrak{A}, \mathfrak{N})$ such that

$$
Q \psi={ }^{A} \alpha U \text { and } \psi V=R \alpha^{I} .
$$

Proof. Suppose there exists $\alpha: \gamma \rightarrow \delta$ so that (3.1) holds. Then, for $X \in \mathscr{M}$

$$
\Gamma(X) \psi=R \gamma(X) Q \psi=R \gamma(X)^{\Lambda} \alpha U=R \alpha^{I} \delta(X) U=\psi V \delta(X) U=\psi \Delta(X) .
$$

Hence $\psi$ is a morphism $\Gamma \rightarrow \Delta$.

Conversely, pick $(\lambda, i) \in \Delta \times I$ such that $p_{\lambda i}$ is invertible and let $\beta: \gamma_{i \lambda} \rightarrow \gamma$ and $\phi: \delta_{i \lambda} \rightarrow \delta$ be the equivalences between $\gamma, \delta$ and the representations induced by $\Gamma$ and $\Delta, i, \lambda$. Now

$$
\Gamma\left(x p_{\lambda i}^{-1} ; i, \lambda\right) \psi=\psi \Delta\left(x p_{\lambda i}^{-1} ; i, \lambda\right)
$$

so that $\psi$ maps $C_{i \lambda}$ into $D_{i \lambda}$; denote by $\alpha_{i \lambda}$ the restriction and corestriction of $\psi$ to $C_{i \lambda}$ and $D_{i \lambda}$. Then, if we write $\alpha=\beta^{-1} \alpha_{i \lambda} \phi$,

$$
\begin{aligned}
\gamma(x) \alpha=\gamma(x) \beta^{-1} \alpha_{i \lambda} \phi & =\beta^{-1} \gamma_{i \lambda}(x) \alpha_{i \lambda} \phi=\beta^{-1} \alpha_{i \lambda} \delta_{i \lambda}(x) \phi \\
& =\beta^{-1} \alpha_{i \lambda} \phi \delta(x)=\alpha \delta(x)
\end{aligned}
$$

for each $x \in \mathfrak{A}$. Hence $\alpha$ is a morphism $\gamma \rightarrow \delta$.

For each $j \in I, R_{j}=\bar{R}_{j} \beta$ where $\bar{R}_{j}$ is the corestriction of $\Gamma\left(p_{\lambda i}^{-1} ; j, \lambda\right)$ to $C_{i \lambda}$. 
Therefore

Hence $R \alpha^{I}=\psi V$.

$$
\begin{aligned}
R_{j} \alpha=\bar{R}_{j} \beta \alpha=\bar{R}_{j} \alpha_{i \lambda} \phi & =\left(\operatorname{corest} \Gamma\left(p_{\lambda i}^{-1} ; j, \lambda\right) \psi\right) \phi \\
& =\left(\operatorname{corest} \psi \Delta\left(p_{\lambda i}^{-1} ; j, \lambda\right)\right) \phi \\
& =\psi\left(\operatorname{corest} \Delta\left(p_{\lambda i}^{-1}: j, \lambda\right)\right) \phi \\
& =\psi \bar{V}_{j} \phi=\psi V_{j} .
\end{aligned}
$$

Similarly $Q \psi={ }^{A} \alpha U$ so that (3.1) is satisfied.

CoRollaRY 3.3. Two representations $\Gamma=[\gamma ; Q, R]$ and $\Delta=[\delta ; U, V]$ of a Munn ring $\mathscr{M}$ are equivalent if and only if there is an equivalence $\alpha: \gamma \rightarrow \delta$ and an isomorphism $\psi$ : Codom $Q \rightarrow \operatorname{Codom} U$ such that

$$
U={ }^{\Lambda}\left(\alpha^{-1}\right) Q \psi, \quad V=\psi^{-1} R \alpha^{I} .
$$

Proof. Suppose that $\alpha, \psi$ exist with these properties then, by the theorem, $\psi$ is an equivalence $\Gamma \rightarrow \Delta$.

Conversely, if $\psi$ is an equivalence $\Gamma \rightarrow \Delta$, let $\alpha$ be defined as in the proof of the theorem. Since $\psi$ is an isomorphism, so is $\alpha_{i \lambda}$ and hence so is $\alpha$.

If $[\gamma ; Q, R]$ and $[\delta ; U, V]$ are representations of $\mathscr{M}(\mathfrak{U} ; I, \Lambda ; P)$ and $\alpha, \psi$ are as in the statement of Theorem 3.2 then we say that $\psi$ extends the morphism $\alpha: \gamma \rightarrow \delta$.

THEOREM 3.4. Let $\Gamma=[\gamma ; Q, R]$ and $\Delta=[\delta ; U, V]$ be basic representations of a Munn ring $\mathscr{M}=\mathscr{M}(\mathfrak{A} ; I, \Lambda ; P)$ over a ring $\mathfrak{R}$ with identity. For each morphism $\alpha: \gamma \rightarrow \delta$ there is a unique morphism $\psi: \Gamma \rightarrow \Delta$ which extends $\alpha$.

Proof. We have

Hence, since $V$ is monic,

$$
Q R \alpha^{I}={ }^{\Lambda} \alpha U V
$$

$$
\operatorname{Ker} Q \subseteq \operatorname{Ker} Q R \alpha^{I}=\operatorname{Ker}^{A} \alpha U V=\operatorname{Ker}^{\Lambda} \alpha U \text {. }
$$

Because $Q$ is epic, there is thus a unique $\psi$ : Codomain $Q \rightarrow$ Codomain $U$ such that $Q \psi={ }^{\Lambda} \alpha U$. But then

$$
Q \psi V={ }^{A} \alpha U V=Q R \alpha^{I}
$$

and so, since $Q$ is epic, we also have $\psi V=R \alpha^{I}$. Thus $\psi$ is a morphism $\Gamma \rightarrow \Delta$ which extends $\alpha$ and is the unique such.

COROLlaRY 3.5. Basic extensions of equivalent representations of $\mathfrak{A}$ are equivalent.

Theorem 3.4 sets up a functor from the category of proper representations of $\mathfrak{A}$ to the category of basic representations of $\mathscr{M}=\mathscr{M}(\mathfrak{A} ; I, \Lambda ; P)$. On the other hand Theorem 2.2 describes a functor from the category of representations, and thus from the category of basic representations, of $\mathscr{M}$ to the category of proper representations of $\mathfrak{A}$. 
THEOREM 3.6. Let $\mathscr{M}=\mathscr{M}(\mathfrak{U} ; I, \Lambda ; P)$ be a Munn ring and let $\mathfrak{N}$ be a ring with identity. Then the category $B(\mathscr{M}, \mathfrak{R})$ of basic representations of $\mathscr{M}$ over $\mathfrak{R}$ is equivalent to the category $P(\mathfrak{A}, \mathfrak{R})$ of proper representations of $\mathfrak{A}$ over $\mathfrak{A}$.

Proof. Pick $(\lambda, i) \in \Lambda \times I$ such that $p_{\lambda i}$ is invertible and let $P$ denote the functor which associates with $\Gamma: \mathscr{M} \rightarrow \operatorname{Hom}(C, C)$ the representation $\gamma_{i \lambda}: \mathfrak{A} \rightarrow$ Hom $\left(C_{i \lambda}, C_{i \lambda}\right)$ of $\mathfrak{A}$ induced by $\Gamma, i, \lambda$.

For each $\gamma \in P(\mathfrak{A}, \mathfrak{R})$ pick and fix an epic $Q^{\gamma}$ and a monic $R^{\gamma}$ such that $Q^{\gamma} R^{\gamma}=$ $\gamma(P)$. Let $B$ denote the functor which associates $\left[\gamma ; Q^{\gamma}, R^{\gamma}\right]$ with $\gamma$ and which associates with $\alpha: \gamma \rightarrow \delta$ the unique morphism $\psi=B(\alpha)$ produced by Theorem 3.4. (The uniqueness of $\psi$ ensures that $B$ is a functor.)

For each $\gamma \in P(\mathfrak{A}, \mathfrak{N})$ let $\eta_{\gamma}$ be the composite of $\gamma\left(p_{\lambda i}^{-1}\right)$ and the corestriction of $Q_{\lambda}^{\gamma}$ to its image $C_{i \lambda}^{\gamma}$. Then, by the proof of Theorem 2.3, $\eta_{\gamma}$ is an equivalence $\gamma \rightarrow \gamma_{i \lambda}$. Suppose $\alpha: \gamma \rightarrow \delta$ and let $\psi=B(\alpha)$. Then $\alpha_{i \lambda}=P(\psi)$ is the restriction and corestriction of $\psi$ to a morphism $C_{i \lambda}^{\gamma} \rightarrow C_{i \lambda}^{\delta}$.

Consider the diagram

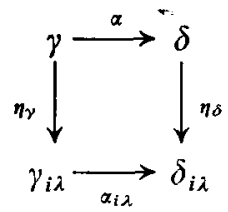

Then $\eta_{,} \alpha_{i \lambda}$ is the corestriction of $\gamma\left(p_{\lambda i}^{-1}\right) Q_{\lambda}^{\gamma} \psi$ to $C_{i \lambda}^{\delta}$

$$
\begin{aligned}
& =\text { corestriction } \gamma\left(p_{\lambda i}^{-1}\right) \alpha Q_{\lambda}^{\delta} \text { to } C_{i \lambda}^{\delta} \\
& =\text { corestriction } \alpha \delta\left(p_{\lambda i}^{-1}\right) Q_{\lambda}^{\delta} \text { to } C_{i \lambda}^{\delta} \\
& =\alpha \eta_{\delta} .
\end{aligned}
$$

Hence $\eta=\left\{\eta_{\gamma}: \gamma \in P(\mathfrak{A}, \mathfrak{R})\right\}$ is a natural equivalence from the identity functor on $P(\mathfrak{H}, \mathfrak{R})$ to $P B$.

Let $\Gamma$ be a basic representation of $\mathscr{M}$ and let $Q^{T}$ be such that $Q_{\mu}^{\Gamma}=$ restriction of $\Gamma(e ; i, \mu)$ to $C_{i \lambda}$ and let $R^{r}$ be such that $R_{j}^{r}=$ corestriction of $\Gamma\left(p_{\lambda i}^{-1} ; j, \lambda\right)$ to $C_{i \lambda}$. Then, as in the proof of Theorem 2.3, $\Gamma=\left[\gamma_{i \lambda} ; Q^{\Gamma}, R^{\Gamma}\right]$. By Theorem 3.4, there is a unique morphism $\psi_{\Gamma}: \Gamma \rightarrow B\left(\gamma_{i \lambda}\right)=B P(\Gamma)$ for each $\Gamma \in B(\mathscr{H}, \mathfrak{R})$. Because of the uniqueness of $\psi$ in Theorem 3.4,

$$
\left\{\psi_{\Gamma}: \Gamma \in B(\mathscr{M}, \mathfrak{R})\right\}
$$

is a natural equivalence from the identity functor on $B(\mathscr{M}, \mathfrak{R})$ to $B P$. Hence the categories $B(\mathscr{M}, \mathfrak{N})$ and $P(\mathfrak{A}, \mathfrak{N})$ are equivalent.

Theorem 3.6 and Proposition 1.5 combine to give the following theorem which contains the results of [1], Theorems 5.48, 5.50, 5.51.

THEOREM 3.7. Let $\Gamma=[\gamma ; Q, R]$ be a representation of a Munn ring $\mathscr{M}=$ $\mathscr{M}(\mathfrak{A} ; I, \Lambda ; P)$ over a ring $\mathfrak{N}$ with identity. Then 
(i) there is a sequence

$$
\Gamma_{1} \rightarrow \Gamma_{2} \rightarrow \Gamma
$$

of subrepresentations of $\Gamma$ where $\Gamma_{1}$ and $\Gamma / \Gamma_{2}$ are null and

$$
\Gamma_{2} / \Gamma_{1}=B(\gamma)
$$

(ii) if $\Gamma$ is proper and $\gamma$ is indecomposable so is $\Gamma$.

(iii) if $\Gamma$ is basic then so is each direct summand of $\Gamma$ and $\Gamma=\Pi B\left(\gamma_{i}\right)$ if and only if $\gamma=\Pi \gamma_{i}$.

(iv) $\Gamma$ is irreducible if and only if it is basic and $\gamma$ is irreducible.

(v) if $\mathfrak{R}$ is a field and codomain $Q$ is finite dimensional then the non null irreducible constituents of $\Gamma$ ( $\mathrm{cf}[3]$, page 153) are the basic extensions of the irreducible constituents of $\gamma$.

Proof. (i) By Proposition 1.5, there is a sequence of subrepresentations

$$
\Gamma_{1} \rightarrow \Gamma_{2} \rightarrow \Gamma
$$

where $\Gamma_{1}$ and $\Gamma / \Gamma_{2}$ are null and $\Gamma_{2} / \Gamma_{1}$ is basic. Hence we need only show that $\Gamma_{2} / \Gamma_{1}$ extends $\gamma$.

Let $(\lambda, i) \in A \times I$ be such that $p_{\lambda i}$ is invertible and let $P$ be the functor in Theorem 3.6. Then, since the sequence

$$
0 \rightarrow \Gamma_{1} \rightarrow \Gamma \rightarrow \Gamma / \Gamma_{1} \rightarrow 0
$$

is exact, so is the sequence

$$
0 \rightarrow P\left(\Gamma_{1}\right) \rightarrow P(\Gamma) \rightarrow P\left(\Gamma / \Gamma_{1}\right) \rightarrow 0 .
$$

Because $\Gamma_{1}$ is null, $P\left(\Gamma_{1}\right)=0$ and therefore $P\left(\Gamma / \Gamma_{1}\right)=P(\Gamma)$. The sequence

$$
0 \rightarrow \Gamma_{2} / \Gamma_{1} \rightarrow \Gamma / \Gamma_{1} \rightarrow \Gamma / \Gamma_{2} \rightarrow 0
$$

is also exact and so gives rise to an exact sequence in $P(\mathfrak{A}, \mathfrak{R})$. Since $\Gamma / \Gamma_{2}$ is null we then have $P\left(\Gamma / \Gamma_{1}\right)=P\left(\Gamma_{2} / \Gamma_{1}\right)$ and so, because $\Gamma$ extends $\gamma, \Gamma_{2} / \Gamma_{1}$ extends $\gamma$.

The other four parts of the theorem follow similarly.

The results in Theorem 3.7 can be proved directly, that is without using homological arguments. We indicate how this can be done in the next section.

\section{Appendix A}

In this section we describe briefly how the results of Theorem 3.7 can be proved without explicitly using the functors of Proposition 1.5 and Theorem 3.6. We shall assume that the ring $\Phi$, over which the representations are being taken, is a field. 
(i) Suppose that $\Gamma=[\gamma ; Q, R]: \mathscr{M} \rightarrow \operatorname{Hom}(C, C)$ is a representation of $\mathscr{M}$ over $\Phi$. Then we can write $C=C_{1} \oplus C_{2} \oplus C_{3}$ where $C_{1}=N(\Gamma)=\operatorname{Ker} R$ and $C_{1} \oplus C_{2}=N(\Gamma)+I(\Gamma)=\operatorname{Ker} R+\operatorname{Im} Q$.

Relative to $C_{1} \oplus C_{2} \oplus C_{3}, R$ has the matrix form

$$
\left(\begin{array}{c}
0 \\
R_{1} \\
R_{2}
\end{array}\right)
$$

where $R_{1}$ (and $\left.R_{2}\right)$ is monic and $Q$ has the form $\left(Q_{0} Q_{1} 0\right)$ where $Q_{1}$ is epic. Hence, for each $X \in \mathscr{M}, \Gamma(X)$ has the form

$$
\left[\begin{array}{ccc}
0 & 0 & 0 \\
R_{1} \gamma(X) Q_{0} & R_{1} \gamma(X) Q_{1} & 0 \\
R_{2} \gamma(X) Q_{0} & R_{2} \gamma(X) Q_{2} & 0
\end{array}\right]
$$

where $\left[\gamma ; Q_{1}, R_{1}\right]$ is the basic extension of $\gamma$; (note $Q_{1} R_{1}=Q R=\gamma(P)$ ).

(ii) Suppose that $\gamma: \mathfrak{A} \rightarrow \operatorname{Hom}(A, A)$ is a representation of $\mathfrak{A}$ over $\Phi$ and consider the representation $\Gamma: \mathscr{M} \rightarrow \operatorname{Hom}\left({ }^{A} A,{ }^{A} A\right)$ defined by $\Gamma(X)=\gamma(P X)$; $\left(\Gamma=\left[\gamma ; I_{A}, P\right]\right)$. If $\gamma=\gamma_{1} \oplus \gamma_{2}$ then $\Gamma=\Gamma_{1} \oplus \Gamma_{2}$ where $\Gamma_{i}(X)=\gamma_{i}(P X)$, $i=1,2$. The basic component of $\Gamma$ is the basic extension of $\gamma$ while the basic component of $\Gamma_{i}$ is the basic extension of $\gamma_{i}, i=1,2$. Hence $B(\gamma)=B\left(\gamma_{i}\right) \oplus B\left(\gamma_{2}\right)$.

A similar situation prevails with regard to reduction. The method outlined in (ii) is an obvious adaptation of the method used by Lallement and Petrich [4] to find irreducible representations.

\section{Appendix B}

Clifford [1] shows that every finite dimensional representation $\Gamma$ of a completely 0 -simple semigroup $\mathscr{M}^{0}(G ; I, A ; P)$, over a field $\Phi$, is equivalent to one of the form

$$
\Gamma^{\prime}(a ; i, \lambda)=\left[\begin{array}{ll}
\gamma\left(p_{1 i} a p_{\lambda 1}\right) & \gamma\left(p_{1 i} a\right) \bar{Q}_{\lambda} \\
\bar{R}_{i} \gamma\left(a p_{\lambda 1}\right) & \bar{R}_{i} \gamma(a) \bar{Q}_{\lambda}
\end{array}\right]
$$

where $\bar{Q}_{1}=0=\bar{R}_{1}$ and $\bar{Q}_{\lambda} \bar{R}_{i}=\gamma\left(p_{\lambda i}-p_{\lambda 1} p_{1 i}\right)$; it is assumed that $p_{11}=e$ the identity of $G$. ([3]. Theorem 5.37).

Let

$$
R=\left(\cdots R_{i} \cdots\right) \quad Q=\left(\begin{array}{c}
\vdots \\
\dot{Q}_{\lambda} \\
\vdots
\end{array}\right)
$$

where

$$
R_{i}=\left[\begin{array}{c}
\gamma\left(p_{1 i}\right) \\
\bar{R}_{i}
\end{array}\right] \text { and } Q_{\gamma}=\left[\gamma\left(p_{i 1}\right) \bar{Q}_{\gamma}\right]
$$


Then

$$
\Gamma^{\prime}(a ; i, \lambda)=R \gamma(A) Q
$$

where $A=(a ; i, \lambda)$, the $I \times \Lambda$ matrix with $a$ in the $i, \lambda^{\text {th }}$ position and zeros elsewhere; by linearity, this extends to $\mathscr{M}(\Phi(G) ; I, \Lambda ; P)$.

Since, by Clifford's theorem quoted above, every finite dimensional representation of $\mathscr{M}(\Phi(G) ; I, \Lambda ; P)$ over $\Phi$ is equivalent to one given by (4.1), it follows that every finite dimensional representation is of the form

$$
\Gamma(X)=R^{\prime} \gamma(X) Q^{\prime}
$$

where $R^{\prime}=B^{-1} R, Q^{\prime}=Q B$ with $B$ invertible over $\Phi$. But

$$
Q^{\prime} R^{\prime}=Q B B^{-1} R=Q R=\gamma(P) .
$$

Hence $\Gamma$ is of the form described by Theorem 2.3.

\section{Appendix C}

Lallement and Petrich [4] have shown that the irreducible representations of a finite 0 -simple semigroup $S=\mathscr{M}^{0}(G ; m, n ; P)$, over a field $\Phi$, can be obtained as follows. Let $\gamma$ be an irreducible representation of $G$ over $\Phi$; then $\gamma$ is finite dimensional, say of dimension $r$. Let $A$ be an irreducible $n r \times n r$ matrix over $\Phi$ such that

$$
A \gamma(P)=\left[\begin{array}{c}
A_{1} \\
0
\end{array}\right]
$$

where $A_{1}$ is $t \times m r$ with row rank $t$. Let

$$
I_{t, n r}=\left[I_{t} 0\right] \text { and } I_{n r, t}=\left[\begin{array}{c}
I_{t} \\
0
\end{array}\right]
$$

Then

$$
\Gamma(X)=I_{t, n r} A \gamma(P X) A^{-1} I_{n r, t}
$$

is an irreducible representation of $S$ which extends $\gamma$.

We may prove this as follows. Let $Q=A^{-1} I_{n r, t}, R=I_{t, n r} A \gamma(P)$. Then

$$
Q R=A^{-1}\left[\begin{array}{cc}
I_{t} & 0 \\
0 & 0
\end{array}\right]\left[\begin{array}{c}
A_{1} \\
0
\end{array}\right]=A^{-1}\left[\begin{array}{c}
A_{1} \\
0
\end{array}\right]=A^{-1} A \gamma(P)=\gamma(P)
$$

so that $\Gamma$ is of the form given by Theorem 2.3. Further, since $A$ is invertible $Q$ is epic and, by the choice of $A, R$ is monic. Hence, by Theorem 3.7, $\Gamma$ is irreducible. 


\section{Appendix D}

Munn [9] has shown that $S=\mathscr{M}^{\circ}(G ; m, n ; P)$ has semisimple algebra over $\Phi$ if and only if $m=n$ and $P$ is invertible over $\Phi(G)$. If this is the case then he shows that the irreducible representations of $S$ over $\Phi$ are the representations of the form $X-W \rightarrow \gamma(P X)$ where $\gamma$ is an irreducible representation of $G$, say of degree $r$. This follows easily from the theory given here. Since $P$ is invertible over $\Phi(G), \gamma(P)$ is invertible over $\Phi$. Hence, in the factorisation, $\gamma(P)=I_{n r} \gamma(P)=Q R$, $Q$ is epic and $R$ is monic. By Theorem 3.7, this means the representation $X-W \rightarrow \gamma(P X)$ is irreducible and extends $\gamma$; hence it is, to within equivalence, the only irreducible representation which extends $\gamma$.

\section{References}

[1] A. H. Clifford, 'Matrix representations of completely simple semigroups', Amer. J. Math. 64 (1942) 327-342.

[2] A. H. Clifford, 'Basic representations of completely simple semigroups', Amer. J. Math. 82 (1960) $430-434$.

[3] A. H. Clifford and G. B. Preston, Algebraic theory of semigroups. Vol. 1. (Math. Surveys of the American Math. Soc. 7, Providence, R. I., 1962).

[4] G. Lallement and M. Petrich, 'Irreducible matrix representations of finite semigroups', Trans. Amer. Math. Soc. 139 (1969) 393-412.

[5] D. B. McAlister, 'Basic representations of arbitrary semigroups', Trans. Amer. Math. Soc. 135 (1969) 311-342.

[6] D. B. McAlister, 'Rings related to completely O-simple semigroups', J. Australian Math. Soc. 3 (1971) 257-274.

[7] D. B. McAlister, 'Basic representations of finite semigroups', Proc. Royal Irish Acad. 69 (1970) 97-111.

[8] B. Mitchell, Theory of categories (Academic Press, New York, 1965).

[9] W. D. Munn, 'On semigroup algebras', Proc. Cambridge Phill. Soc. 51 (1955) 1-15.

[10] B. L. Van der Waerden, Modern Algebra. Vol. 2 (Fredrick Ungar Publishing Co., New York, 1950).

Department of Mathematics

Northern Illinois University

De Kalb

Illinois 60115 